\title{
THE HISTORY OF RESEARCH OF THE OPHTHALMIC ASPECTS OF HYPERTENSION
}

\author{
Aleksandra Krasińska ${ }^{1}$, Agata Brązert ${ }^{1}$, Jarosław Kocięcki ${ }^{1}$
}

\begin{abstract}
The awareness of the widespread influence of hypertension on various organ systems is ever increasing. Changes associated with this disease can be observed in the heart, brain, kidneys, but also the organ of vision. These usual microvascular changes are defined as hypertensive retinopathy. During a funduscopic examination, abnormalities such as narrowing of arterioles, symptoms of arteriole and vein intersection, cotton wool spots, intra-retinal exudates, retinal haemorrhages, and in severe cases even swelling of the optic disc and macula. This review presents an overview of the changes at the fundus of the eye that may occur in patients with hypertension, as well as problems with the classification of hypertensive retinopathy over the years, and the development of diagnostic methods in ophthalmology and fundoscopic imaging.
\end{abstract}

Running title: The history of hypertensive retinopathy research

Keywords: hypertension, retinopathy, fundus, ophthalmoscopic examination 


\section{Introduction}

We are currently facing a global epidemic of hypertension, with more than 1 billion people worldwide who are affected by this disease, one of the most important causes of premature mortality in the human population. Furthermore, the awareness of the widespread influence of hypertension on various organ systems is ever increasing. Changes associated with this disease can be observed in the heart, brain, kidneys, but also the organ of vision. Moreover, the eyes are the only place where changes at the vascular level can be non-invasively visualized, often before the patient is diagnosed with hypertension [1]. These usual microvascular changes are defined as hypertensive retinopathy. During a funduscopic examination, abnormalities such as narrowing of arterioles, symptoms of arteriole and vein intersection, cotton wool spots, intra-retinal exudates, retinal haemorrhages, and in severe cases even swelling of the optic disc and macula. Poorly controlled hypertension further affects the development and deterioration of retinopathy [2]. Hypertension also increases the development of diabetic retinopathy, which is one of the leading causes of blindness among working age population [2]. This review presents an overview of the changes at the fundus of the eye that may occur in patients with hypertension, as well as problems with the classification of hypertensive retinopathy over the years, and the development of diagnostic methods in ophthalmology and fundoscopic imaging.

\section{The beginnings of research}

The possibility of fundus observation, hence examination of all structures in the posterior pole of the eye first appeared in 1851, with the invention of the first ophthalmoscope by Herman von Helmholtz [3]. In turn, in 1859, Liebreich connected the pathological changes on the fundus with arterial hypertension, based on the example of Bright's disease. A further ground-breaking medical discovery, the invention of the sphygmomanometer in 1896, brought the attention of scientists to the aspect of hypertension and fundus vascular changes. In the same year, Albutt classified hypertension as a disease, due to its connection to the development of atherosclerosis. Later, in 1892 and 1898, Marcus Gunn published two reports defining the changes in fundal arterioles as "copper-wires", describing these vessels as much paler compared to the healthy eye, with particularly bright, sharp light reflection. These changes were noted in patients with albuminuria, as well as hypertension. The author also brought focus to the arteriole-vein crossings, accompanied by a partial narrowing of vein fragments. However, this symptom, known nowadays as arteriovenous nicking, did not gain significant attention at the time [4]. Several decades later, in 1930, Friedenwald connected vascular changes with atherosclerosis [5]. The concept of vein narrowing returned in 1936 when Jensen reported that atherosclerosis is also accompanied by venous changes. In some of the patients he studied, venous alterations even surpassed those observed in the arterioles. Hence, he concluded that the abnormalities in arteriole wall nutrient supply are associated with the pressure exerted by narrowed veins.

Furthermore, in 1937, Ballantyne updated the classification of fundal vascular changes. He differentiated "copper-wires", indicating early atherosclerotic changes, from "silver-wires", which were associated with advanced atherosclerosis. Moreover, he also noted that silver-wires can occur in non-hypertensive patients, most commonly due to obstruction of central retinal vein [4].

\section{Classification of hypertensive retinopathy}

In 1939, the first classification of hypertensive retinopathy was published, designed by Keith, Wagner, and Barker [6]. It consists of four stages. In the first, partial stenosis of retinal arterioles can be observed, with prominent stenosis, arteriovenous nicking, small haemorrhages, and hard exudates characteristic for the second stage. In turn, the third stage was recognized based on the presence of cotton wool spots and flame-shaped haemorrhaging. Finally, the fourth stage correlated with severe hypertension, with swelling of the optic disc observed at the fundus. The authors also showed that 3-year survival for stage 1 amounted to $70 \%$, while for stage 4 it was only $6 \%$. They further emphasized the value of the ophthalmoscopic examination, as the only diagnostic procedure allowing to directly observe microvascular changes.

The next couple of years did not bring significant changes to the definition of hypertensive retinopathy. The next attempt at classification of this disease took place in 1947 when Wagener, Clay, and Gipner tried to improve the previously designed scale. However, their efforts were unsuccesfull [7].

Scheie modified the Keith-Wagner-Barker scale in 1953, adding another stage, zero, indicating no pathological changes in the fundus. The first stage was accompanied by minor arteriole stenosis. In the second stage, arteriole stenosis and irregularity were seen, as well as arteriovenous nicking. In the third degree, intra-retinal haemorrhaging and exudations, both soft and hard, were also visible. Finally, the last stage was characterized by optic disc swelling. The author also noted that intensification of changes in fundal vessels corresponded with the duration of hypertension. Furthermore, he indicated a correlation of earlier occurrence of said changes with significantly elevated systemic pressure.

In 1957, Leishman emphasized the importance of the presence or absence of atherosclerosis in fundal arterioles in hypertension, as it affected the picture analysed during the examination. He also demonstrated that sclerosis of fundal arterioles can 
also occur during the natural aging process. Furthermore, the author brought attention to factors affecting such appearance of the vessels, namely: diabetes, eye injury, and degenerative diseases. He created a classification, consisting of seven stages, that proved to be too complicated to be used in everyday clinical practice. However, other authors agreed that the presence or absence of sclerotic arterioles is an important factor in the fundoscopic examination of hypertensive patients.

In the following years, the applicability of the common scales in clinical practice was a subject of continuous critique. The vocal opponents mostly criticised the two main stages of Keith-Wegner-Barker and Scheie scales. Their main problem concerned the barely noticeable differences between these stages during the ophthalmoscopic examination, which made proper classification almost impossible. Furthermore, the stages did not properly correlate with the severity of hypertension. Yet another group of scientists brought attention to the fact that the studies were performed on groups with uncontrolled hypertension, which caused the presence of fundal vasculature changes $[7,8]$.

In 1966, an updated version of the Keith-Wegener-Barker study was published. In this publication, the focus was placed on the degree of arteriole stenosis, the definition of this occurrence is focal or systemic, as well as the presence of sclerosis. This allowed for more objective clinical evaluation, hence the easier classification of hypertensive retinopathy stage [7].

In 1970 , a paper on the survivability of the patients with hypertension was published, the studies, conducted in 1952-1967, showed increased survival compared to the initial evaluation of Keith-Wegener-Barker: $71 \%, 51 \%, 31 \%$, and $21 \%$ in stages I-IV respectively. This increase in survival among patients was suggested to be caused by better clinical management of hypertension compared to 1939 [9].

In turn, a large prospective study conducted in 1978 in Gothenburg, a group of 855 patients aged 4-10 were evaluated. It was noted that arteriole changes are the most sensitive ophthalmoscopic examination marker, with stenosis further emphasising the presence of hypertension. This study also reported that neither arteriovenous nicking nor the increased reflectiveness of arterioles is characteristic for hypertension, as they are also observed in healthy population [10].

\section{Modern world advancements in fundus examination}

Due to developments in ophthalmology, new methods of fundal imaging were introduced to clinical practice. In 1886, Jackman and Webster were the first to publish a picture of the fundus of the eye. However, the visibility was limited to the fundal reflection and faint borders of the optic disc. Hence,
Howe and Starr are considered as the real pioneers of fundal photography, as they were the first that, as a part of their studies conducted in $1886-1888$, took a picture of the fundus allowing to discern its anatomical structures. In 1907, Friedrich Dimmer, together with Zeiss Jena, built the first fundus camera. Unfortunately, the size and price of the apparatus did not allow for mass production. In 1911, Gullstrant invented the first slit lamp that introduced a significant advantage in patient examination. In 1925, Nordenson and Zeiss introduced the first mass-produced fundus camera. Soon afterward, an atlas of fundus pictures was published, taken using a Zeiss apparatus created by Arthur Bedell. In turn, the fundus camera on which modern photographic documentation is based on, was created in 1955 [11]. Over the years, a new method of ophthalmic diagnostics was introduced - fluorescein angiography. Fluorescein, a dye, now commonly used in ophthalmology, was synthesised by Paul Ehrlich in 1882. While numerous scientists attempted its use it in ophthalmic diagnostics, it wasn't until 1960 when two students from Indiana University managed to apply fluorescein angiography in fundoscopic examination [12]. This method is based on the injection of dye into patients' bloodstream, allowing to visualise the contents of the vessels using black and white picture taken using a fundus camera. It is currently used for diagnostics of several diseases, such as diabetes, age-related macular degeneration, as well as eye-associated hypertrophy.

In 1996, Hayreh also openly criticised the use of the Keith-Wegener-Barker scale in the clinical practice. He argued that a vastly superior method could be based on describing the changes observed in the fundus during the examination, complemented with fluorescein angiography. This, in his opinion, could bring significant ease to control visits and notice changes associated with disease progression. He also brought attention to the fact that not only hypertension but also lower-than-normal blood pressure leads to optic nerve fibre damage. This, in consequence, can lead to vision disturbances. Furthermore, overuse of blood pressure-lowering drugs, particularly in night hours when a natural drop of pressure occurs, can also lead to optic nerve fibre damage [13].

In the further years, several studies confirmed the correlation between hypertension and fundal retinopathy symptoms [14]. In 2001, Wong proved that arteriole stenosis and arteriovenous nicking occur in chronic hypertension, and are good markers for evaluation of hypertension severity [14]. He also noted that other changes such as retinal haemorrhages, cotton wool spots, and microaneurysms, could be better as markers for estimation of hypertension severity, as they are not associated with the system-wide occurrence of this disease. Presence of these symptoms in the eye fundus was connected 
to a higher risk of stroke occurrence, compared to patients in which such changes did not occur [8]. In a later work, published in 2004, the same author proved that the presence of arteriole stenosis and arteriovenous nicking in people without previously recognized hypertension might suggest a pre-hypertensive state. He also underlined the importance of fundus examination in patients with hypertension. However, according to data presented in the work, the direct examination is unobjective, encouraging the use of a fundus camera [8]. Two years later, Wong revised his opinion to perform routine ophthalmologic examinations in all hypertensive patients. In turn, he advised such procedures only in those exhibiting other risk factors, e.g. diabetes or blurry vision symptoms [1].

All of these conclusions led, in 2005, to the creation of a simplified hypertensive retinopathy scale, in which three stages of severity were indicated (mild, intermediate and severe). Each of the stage contained a detailed description of fundal changes, as well as their correlation with systemic symptoms and suggestions for adequate disease management [15]. In the same year, Grosso took up the topic of white coat hypertension and its correlation to the presence of hypertensive retinopathy. According to his work, if retinopathy symptoms occur in white coat hypertension patients, the introduction of blood pressure-lowering drugs should be considered [16].

\section{The OCT breakthrough}

In the modern history of ophthalmology, one of the most ground-breaking moments concerned the invention of optical coherent tomography (OCT) of the fundus. This device was first designed by Swanson in 1992, resulting in a publication of an OCT scan atlas as early as 1996 [17]. This examination allowed us to obtain spatial retinal scans, which makes it possible to evaluate its posterior region on a microscopic level. OCT makes it possible to image the retina including the thickness of its nerve fibres, blood vessels and the optic nerve, in an objective way, as well as to analyse and compare previous scans, enabling trend analysis [18] The fast spread of this method's use was mostly caused by the ease of scanning, as well as the its contactlessness and painlessness. Currently, it is used to monitor optic nerve damage in glaucoma, as well as exudative age-related macular degeneration. In 2014, a three-stage scale was created based on OCT scans, correlated with best corrected visual acuity. It was proven that the OCT image of retinopathy sub-retinal fluid presence and increased choroid thickness, correlated directly with the severity of hypertension, as well as with impaired visual acuity [19]. Before, fluorescein angiography was the most popular method of blood vessels imaging. However, this method requires contrast administration, which can result in unwanted side effects. Hence, the invention of angio - OCT by Barron in 2005, later improved by Mariampillai in 2008 is considered yet another breakthrough in imaging [20]. This examination allows for the visualisation of vessels in a non-invasive manner. This is particularly important in age-related macular degeneration diagnostics, associated with the presence of neovascular membrane. This is currently one of the most prospective clinical applications of OCT.

\section{Conclusions}

The research on systematisation of knowledge and classification of hypertensive retinopathy is still ongoing. New methods of imaging, their constantly improving quality and the possibility to visualise even the smallest details all improve the ability of clinicians to improve the methods of fundus change visualisation in hypertension patients. Significant hopes are associated with the development of angio-OCT, as well as software for the interpretation of this method's results. Unfortunately, the current availability of this highly specialised equipment in clinics is still limited due to significant purchase cost. However, it is possible that, in the future, computer imaging will completely replace visual examination, while clinicians will be able to fully objectively interpret the results using proprietary equipment and software.

\section{Ethical approval}

The conducted research is not related to either human or animal use.

\section{Acknowledgments}

Not applicable.

\section{Corresponding author}

Aleksandra Krasińska, Department of Ophthalmology, Poznan University of Medical Sciences, Szamarzewskiego 84, 60-569, Poznań, Poland, e-mail: alex.krasinska@gmail.com.

\section{Conflict of interest statement}

The authors declare they have no conflict of interest.

\section{References}

1. Wong TY, Mitchell P. Hypertensive Retinopathy. N Engl J Med. 2004;351:2310-7; DOI:10.1056/NEJMra032865

2. Bhargava M, Ikram MK, Wong TY. How does hypertension affect your eyes. J Hum Hypertens. 2012;26:71-83; DOI:10.1038/jhh.2011.37.

3. Pearce JMS. The ophthalmoscope: Helmholtz's Augenspiegel. Eur Neurol. 2009;61:244-9; DOI:10.1159/000198418.

4. Wise GN. Retinal Arteriosclerosis Secondary to Vein Obstruction. AMA Arch Ophthalmol. 1959;61:766-79; DOI:10.1001/ archopht.1959.00940090768013.

5. Nilsson PM, Cifkova R, Kjeldsen SE, Mancia G. European Society of Hypertension Scientific Newsletter: Update on Hypertension Management: Prevention of type 2 diabetes mellitus with antihypertensive drugs. J Hypertens. 2006;24:2478-82; DOI:10.1097/HJH.0b013e328010b96f.

6. Wong TY, Klein R, Klein BEK, Tielsch JM, Hubbard L, Nieto FJ. Retinal microvascular abnormalities and their relationship with hypertension, cardiovascular disease, and mortality. Surv Ophthalmol. 2001;46:5980; DOI:10.1016/S0039-6257(01)00234-X.

7. Walsh JB. Hypertensive Retinopathy: Description, Classification, and Prognosis. Ophthalmology. 1982;89:1127-31; DOI:10.1016/ S0161-6420(82)34664-3.

8. Wong TY, Klein R, Couper DJ, Cooper LS, Shahar E, Hubbard LD, Wofford MR, Sharrett AR. Retinal microvascular abnormalities and in- 
cident stroke: The Atherosclerosis Risk in Communities Study. Lancet. 2001;358:1134-40; DOI:10.1016/S0140-6736(01)06253-5.

9. Breckenridge A, Dollery CT, Parry EH. Prognosis of treated hypertension. Changes in life expectancy and causes of death between 1952 and 1967. Q J Med. 1970;39:411-29; DOI:10.1093/oxfordjournals.qjmed. a067217.

10. Svärdsudd K, Wedel H, Aurell E, Tibblin G. Hypertensive Eye Ground Changes: Prevalence, Relation to Blood Pressure and Prognostic Importance The Study of Men Born in 1913. Acta Med Scand. 1978;204:15967; DOI:10.1111/j.0954-6820.1978.tb08418.x.

11. Bennett T. Milestones In Opthalmic Imaging. J Ophthalmic Photogr. 2019;41:7.

12. Marmor MF, Ravin JG. Fluorescein angiography: Insight and serendipity a half century ago. Arch Ophthalmol. 2011;129:943-8; DOI:10.1001/ archophthalmol.2011.160.

13. Hayreh SS. Systemic arterial blood pressure and the eye. Eye. 1996;10:528; DOI:10.1038/eye.1996.3.

14. Wong TY, Hubbard LD, Klein R, Marino EK, Kronmal R, Sharrett AR, Siscovick DS, Burke G, Tielsch JM. Retinal microvascular abnormalities and blood pressure in older people: The Cardiovascular Health Study. Br J Ophthalmol. 2002;86:1007-13; DOI:10.1136/bjo.86.9.1007.

15. Wong TY, McIntosh R. Systemic associations of retinal microvascular signs: A review of recent population-based studies. Ophthalmic Physiol Opt. 2005;25:195-204; DOI:10.1111/j.1475-1313.2005.00288.x.

16. Grosso A, Veglio F, Porta M, Grignolo FM, Wong TY. Hypertensive retinopathy revisited: Some answers, more questions. Br J Ophthalmol. 2005;89:1646-54; DOI:10.1136/bjo.2005.072546.

17. Swanson EA, Fujimoto JG. The ecosystem that powered the translation of OCT from fundamental research to clinical and commercial impact [Invited]. Biomed Opt Express. 2017;8:1638; DOI:10.1364/ boe.8.001638.

18. Gabriele ML, Wollstein G, Ishikawa H, Kagemann L, Xu J, Folio LS, Schuman JS. Optical coherence tomography: History, current status, and laboratory work. Investig Ophthalmol Vis Sci. 2011;52:2425-36 DOI:10.1167/iovs.10-6312.

19. Ahn SJ, Woo SJ, Park KH. Retinal and choroidal changes with severe hypertension and their association with visual outcome. Investig Ophthalmol Vis Sci. 2014;55:7775-85; DOI:10.1167/iovs.14-14915.

20. Gao SS, Jia Y, Zhang M, Su JP, Liu G, Hwang TS, Bailey ST, Huang D. Optical coherence tomography angiography. Investig Ophthalmol Vis Sci. 2016;57:27-36; DOI:10.1167/iovs.15-19043. 